\title{
Oscilação genética em populações submetidas a métodos de seleção tradicionais e associados a marcadores moleculares
}

\author{
Paulo Luiz Souza Carneiro ${ }^{1}$, Carlos Henrique Mendes Malhado ${ }^{1}$, Ricardo Frederico \\ Euclydes $^{2}$, Robledo de Almeida Torres ${ }^{2}$, Paulo Sávio Lopes ${ }^{2}$, Antonio Policarpo Souza \\ Carneiro $^{3}$, Elizângela Emídio Cunha ${ }^{4}$
}

\footnotetext{
${ }^{1}$ Departamento de Ciências Biológicas - Universidade Estadual do Sudoeste da Bahia

2 Departamento de Zootecnia - Universidade Federal de Viçosa.

${ }^{3}$ Departamento de Informática - Universidade Federal de Viçosa.

${ }^{4}$ Pós-graduação em Genética e Melhoramento - Universidade Federal de Viçosa.
}

RESUMO - Com o objetivo de avaliar o efeito da oscilação genética em populações sob seleção com diferentes tamanhos efetivos, foram simulados dados utilizando-se o programa GENESYS. A seleção foi praticada durante 20 gerações consecutivas, com base na seleção individual (SI) e nos valores genéticos preditos pelo BLUP Clássico (BLUP) e pelo BLUP Marcadores (BLUPM), com 30 repetições. A matriz de similaridade adotada no BLUP Marcadores foi obtida utilizando-se 100 marcadores moleculares do tipo microssatélite, por meio de um coeficiente de similaridade correspondente à distância euclidiana média para dados quantitativos. Foram simuladas seis populações de seleção, correspondendo a dois tamanhos efetivos $(18,18$ e 66,66) e três sistemas de acasalamento dos reprodutores selecionados (reprodutores acasalados ao acaso, exclusão de irmãos completos e exclusão de irmãos completos e meio-irmãos). O parâmetro avaliado foi o valor fenotípico médio. Observou-se grande variação nos valores fenotípicos obtidos pelos métodos, em razão da oscilação genética, principalmente para a seleção baseada nos valores genéticos preditos pelo BLUP e BLUPM e para as populações com tamanho efetivo menor $(18,18)$. Os resultados sugerem que, em programas de melhoramento que utilizam pequenas populações sob seleção, os resultados podem ser influenciados pela oscilação genética, podendo apresentar grandes variações nos ganhos genéticos.

Palavras-chave: avaliação genética, BLUP, seleção individual

\section{Genetic drift in populations under traditional and marker assisted selection}

\begin{abstract}
The effect of genetic drift in populations under selection with different effective sizes was evaluated using data simulated by the GENESYS program. Selection was applied during 20 generations based on Individual selection (IS) using breeding values predicted by classical best linear unbiased prediction (BLUP) and BLUP associated with molecular markers (BLUPM). The similarity matrix used in BLUPM was obtained by simulation of 100 micro-satellites markers (simple sequence repeats) using a similarity coefficient corresponding to the mean Euclidean distance for quantitative data. Six populations of selection were simulated corresponding to two effective sizes (18.18 and 66.66), and three mating systems of selected sires (random mating, exclusion of half sibs and exclusion of half as well as full sibs). The average phenotypic value was the parameter of evaluation. Large variation on average phenotypic values was due to genetic drift, mainly for BLUP and BLUPM methods and also for populations with small effective sizes. The results indicate genetic drift may cause large variation in genetic gains by selection in breeding programs designed for small populations.
\end{abstract}

Key Words: BLUP, individual selection, genetic evaluation

\section{Introdução}

Em grandes populações sob acasalamento ao acaso e na ausência de mutação, migração e seleção, as freqüências gênicas e genotípicas permanecem constantes geração após geração. Entretanto, em pequenas populações, as freqüências gênicas flutuam aleatoriamente ao longo das gerações, como resultado da amostragem de gametas. Estas flutuações aleatórias constituem o processo dispersivo ou oscilação genética.
Nas populações sob acasalamento ao acaso, o processo de oscilação genética é bem entendido, o que não acontece quando se consideram populações sob seleção (Sorensen \& Kennedy, 1983).

As conseqüências da oscilação genética em populações sob acasalamento ao acaso são estudadas em uma população ideal, que apresenta diversas condições restritivas quanto à estrutura de acasalamento das populações, que raramente são encontradas em populações reais. A

Correspondências devem ser enviadas para: pcarneiro@uesb.br 
conexão entre as populações reais e a população ideal nos estudos dos processos dispersivos é feita pelo tamanho efetivo da população (Falconer, 1981). A descrição do processo dispersivo por meio da variância da freqüência gênica ou da taxa de endogamia é realizada transformando-se o número de indivíduos que se acasalam em número efetivo, também denominado tamanho efetivo da população (Falconer, 1981).

Muir (2000) relata que, entre os principais fatores que influem na resposta à seleção, além do tamanho efetivo da população, estão a acurácia e a intensidade de seleção. Freqüentemente, estudos indicam que a resposta ótima à seleção pode ser obtida maximizando-se estes fatores. Entretanto, nem todos os fatores podem ser maximizados simultaneamente. $\mathrm{O}$ aumento na intensidade de seleção, por exemplo, provoca decréscimo no tamanho efetivo da população e, conseqüentemente, na resposta à seleção. Da mesma forma, o aumento na acurácia da seleção pelo uso de índices de família ou BLUP também reduz o tamanho efetivo da população, ocasionando aumento mais rápido na taxa de endogamia (Quinton et al., 1992; Quinton \& Smith, 1995).

Segundo Wei et al. (1996), o índice de seleção e a seleção com base nos valores genéticos preditos pelo BLUP sob modelo animal são formalmente os métodos que proporcionaram a maior resposta à seleção. Assumindo pequena população, Wray \& Hill (1989) computaram respostas assintóticas (taxa de resposta constante) após considerar o efeito "Bulmer” para vários esquemas de índice de seleção. Dekkers (1992) \& Villanueva et al. (1993) estenderam a teoria para a seleção pelo BLUP. Esses autores, entretanto, não consideraram a redução na resposta à seleção, em razão do pequeno tamanho das populações.

Meuwissen \& Woolliams (1994) ressaltaram o fato de que novas metodologias têm sido desenvolvidas no melhoramento animal como forma de aumentar o ganho genético, por exemplo, o BLUP e os núcleos MOET. Entretanto, para esses métodos, as taxas de endogamia são aumentadas concomitantemente, de modo mais intenso em pequenas populações, como no caso de núcleos MOET e de pequenos núcleos para conservação genética. Segundo Muir (2000), o primeiro impacto da endogamia é a perda de alelos decorrente da oscilação genética, que é diretamente proporcional aos níveis de endogamia.

Em estudos recentes realizados no Brasil, o tamanho efetivo de diferentes raças bovinas foi estimado, verificando-se tamanhos efetivos baixíssimos (Faria et al., 2001a, b, c, d, e, f, g). O objetivo neste trabalho foi analisar a dispersão ocorrida nos valores fenotípicos, atribuída à oscilação genética, em 30 repetições, ao longo de 20 gerações de seleção.

\section{Material e Métodos}

Os dados utilizados neste estudo foram obtidos do programa de simulação GENESYS (Euclydes \& Guimarães, 1996), desenvolvido para o compilador FORTRAN. Esse sistema permite simulação de material genético, no qual a expressão de uma característica é determinada pela contribuição de uma série de genes e por efeitos ambientais.

Para possibilitar o desenvolvimento deste trabalho, foi simulado um genoma constituído de uma característica quantitativa com valor de herdabilidade igual a 0,10 . As características desse genoma foram: 200 locos quantitativos polialélicos (oito alelos) cujas freqüências iniciais foram simuladas com base na distribuição uniforme e tiveram valor inicial médio de 0,50 ; os locos foram distribuídos ao acaso em 15 pares de cromossomos autossômicos de tamanhos aleatórios em genoma de 2.000 centimorgans de comprimento; foram simulados apenas efeitos aditivos dos locos quantitativos, segundo a distribuição normal; os locos quantitativos não possuíram desvios de dominância nem interações entre si; as freqüências gênicas iniciais foram iguais em ambos os sexos; a distribuição matemática usada para a simulação das freqüências iniciais dos locos quantitativos nas populações foi a uniforme, com média de 0,50 ; utilizaram-se três efeitos fixos com 10, 4 e 6 níveis, respectivamente; os efeitos de ambiente não-controláveis foram simulados conforme a distribuição normal; para cálculo da matriz de similaridade genética, por meio de marcadores moleculares, foram utilizados 100 marcadores do tipo microssatélite (SSR - Simple Sequence Repeat).

Utilizando-se o genoma supracitado, foi simulada uma população-base de 1.000 indivíduos (500 machos e 500 fêmeas) e, a partir dessa população, foi construída uma população inicial, pela escolha e pelo acasalamento ao acaso de 10 machos e 100 fêmeas, produzindo cinco descendentes por acasalamento, gerando 500 indivíduos. Em seguida, foram formadas as populações de seleção, em um total de seis, correspondendo a dois tamanhos efetivos de população e três sistemas de acasalamento praticados entre os reprodutores selecionados para pais da geração seguinte (Tabela 1 ).

A seleção foi praticada durante 20 gerações consecutivas, utilizando-se a seleção individual - SI e os valores genéticos preditos pelo BLUP Clássico - BLUP (melhor preditor linear não-viesado) e pelo BLUP Marcadores-BLUPM(BLUP utilizando matriz de similaridade genética calculada por meio de marcadores moleculares), com 1, 10 e 30 repetições.

Para as populações com tamanho efetivo 1 (TE1), em cada geração, foram escolhidos cinco machos e 50 fêmeas 
Tabela 1 - Estrutura das populações sob seleção

Table 1 - Structure of populations under selection

\begin{tabular}{|c|c|c|c|}
\hline \multirow[t]{2}{*}{$\begin{array}{l}\text { Tamanho efetivo } \\
\text { Effective size }\end{array}$} & \multicolumn{3}{|c|}{$\begin{array}{c}\text { Sistema de acasalamento } \\
\text { Mating system }\end{array}$} \\
\hline & RAA & EIC & EICMI \\
\hline TE 1 & TE1-RAA & TE1-EIC & TE1-EICMI \\
\hline TE 2 & TE2-RAA & TE2-EIC & TE2-EICMI \\
\hline
\end{tabular}

TE1 - tamanho efetivo 1, TE2 - tamanho efetivo 2, RAA - reprodutores acasalados aleatoriamente, EIC - exclusão de irmãos completos, EICMI - exclusão de irmãos completos e meio-irmãos.

ES1 - effective size 1, ES2 - effective size, RM - random mating of reproducers, EFS - exclusion of full-sibs, EFSHS - exclusion of full-sibs and half-sibs.

e gerados cinco descendentes por acasalamento, totalizando 250 indivíduos (tamanho efetivo de 18,18). Para as populações com tamanho efetivo 2 (TE2), foram escolhidos, a cada geração, 20 machos e 100 fêmeas e gerados cinco descendentes por acasalamento, totalizando 500 indivíduos (tamanho efetivo de 66,66).

O cálculo do tamanho efetivo dessas populações foi feito utilizando-se a fórmula proposta por Wright(1931), citada por Falconer (1981): $\frac{1}{N_{e}}=\frac{1}{4 N_{m}}+\frac{1}{4 N_{t}}$, em que $N_{e}=$ tamanho efetivo da população, $N_{m}=$ número de machos e $N_{f}=$ número de fêmeas.

O efeito da oscilação genética nas diferentes estruturas de populações sob seleção foi avaliado considerando-se o valor fenotípico médio, a endogamia média e a porcentagem de locos fixados desfavoravelmente nos diferentes tamanhos efetivos (TE1 e TE2), os números de repetições (1, 10 e 30) e os sistemas de acasalamento (RAA, EIC e EICMI).

\section{Resultados e Discussão}

Constam nas Figuras de 1 a 9 os valores fenotípicos observados ao longo de 20 gerações de seleção, demonstrando a dispersão ocorrida em 30 repetições. Nesses valores foram considerados: a) três métodos de seleção: seleção individual (SI), seleção baseada nos valores genéticos preditos pelo BLUP Clássico (BLUP) e seleção baseada nos valores genéticos preditos pelo BLUP Marcadores (BLUPM); b) dois tamanhos efetivos de população: 1 (TE1 = 18,18) e $2(\mathrm{TE2}=66,66)$; e c) três sistemas de acasalamento dos reprodutores selecionados: reprodutores acasalados aleatoriamente (RAA), exclusão de irmãos completos (EIC) e exclusão de irmãos completos e meio-irmãos (EICMI).

Analisando as Figuras 1 a 9, observou-se que, de modo geral, as populações de menor tamanho efetivo (TE1) apresentaram maior oscilação que as de maior tamanho efetivo (TE2), independentemente do método de seleção e do sistema de acasalamento utilizados, como observado também

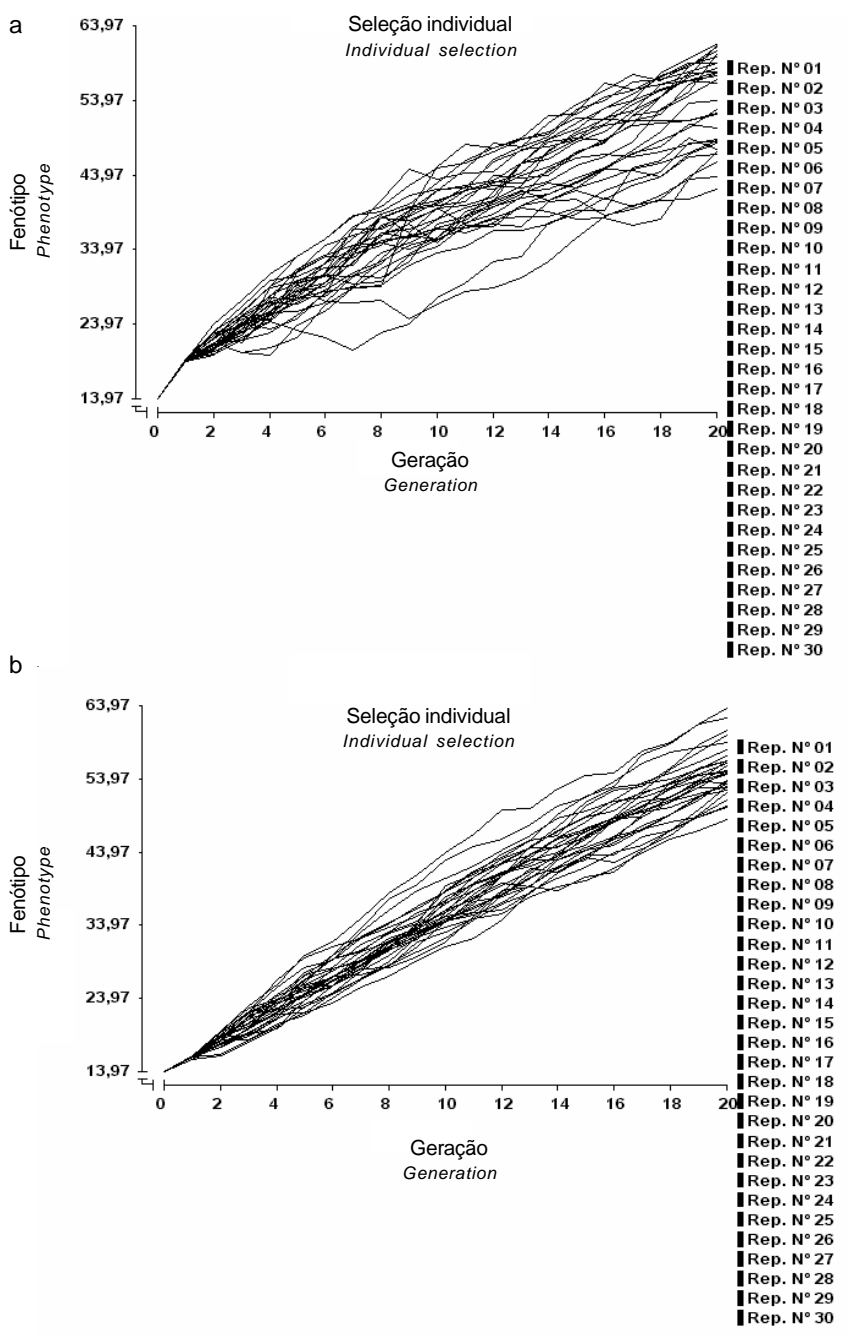

Figura 1 - Valores fenotípicos observados utilizando-se SI com reprodutores acasalados aleatoriamente (RAA), para populações com tamanho efetivo 1 e 2 (a e b), respectivamente.

Figure 1 - Observed phenotypic values using SI with random mating of reproducers (RM), for 1 and 2 effective size of the populations ( $a$ e b), respectively.

por Carneiro et al. (2002), de modo que a oscilação foi mais acentuada para características de baixa herdabilidade e para populações com tamanhos efetivos menores. Essa oscilação, definida por Aggrey et al. (1995) como oscilação genética, nos valores fenotípicos foi conseqüência de mudanças aleatórias na freqüência gênica, causadas principalmente pela amostragem de gametas.

É interessante observar, principalmente nas populações com tamanho efetivo menor (TE1), a grande variação no valor fenotípico após 20 gerações de seleção. Como exemplo, na Figura 4a, algumas repetições chegam a aproximadamente 63,00 unidades de valor fenotípico e outras apenas a 40,00 unidades para o BLUP e, na Figura 7a, repetições chegaram a 58,00 unidades de valor fenotípico e outras a apenas 33,00, para o BLUPM. 


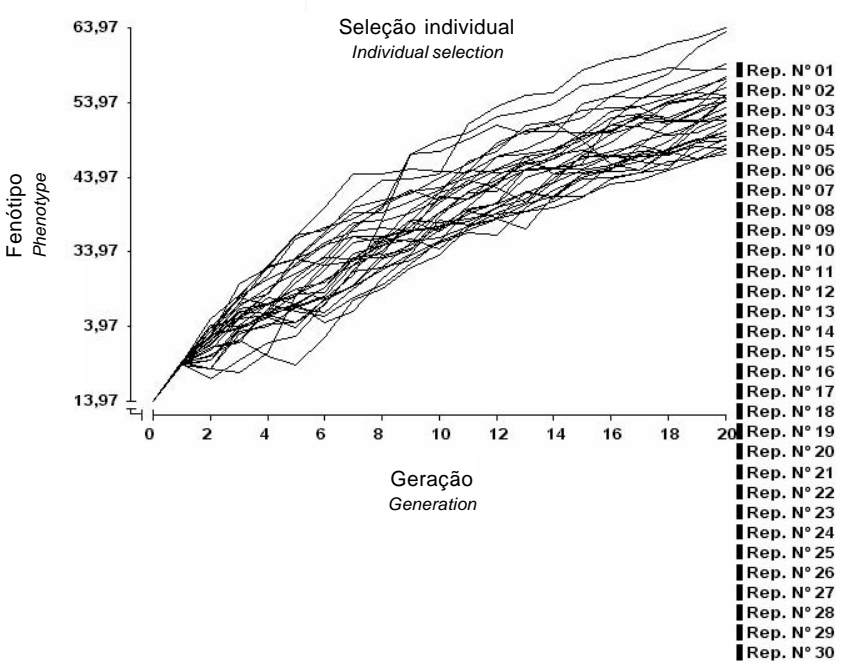

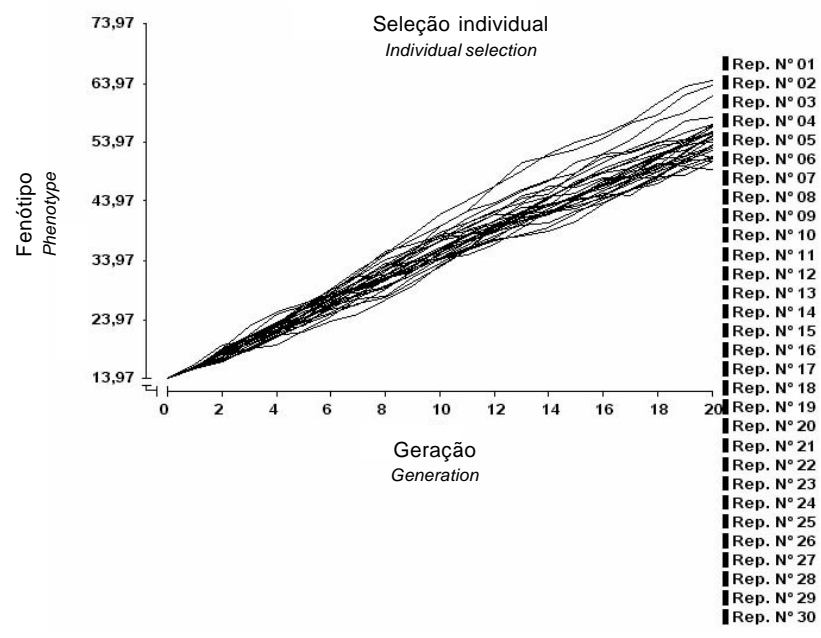

Figura 2 - Valores fenotípicos observados utilizando-se SI com exclusão de irmãos completos (EIC), para populações com tamanho efetivo 1 e 2 (a e b), respectivamente.

Figure 2 - Observed phenotypic values using SI with exclusion of full-sibs; (EFS), for 1 and 2 effective size of the populations (a e b), respectively.
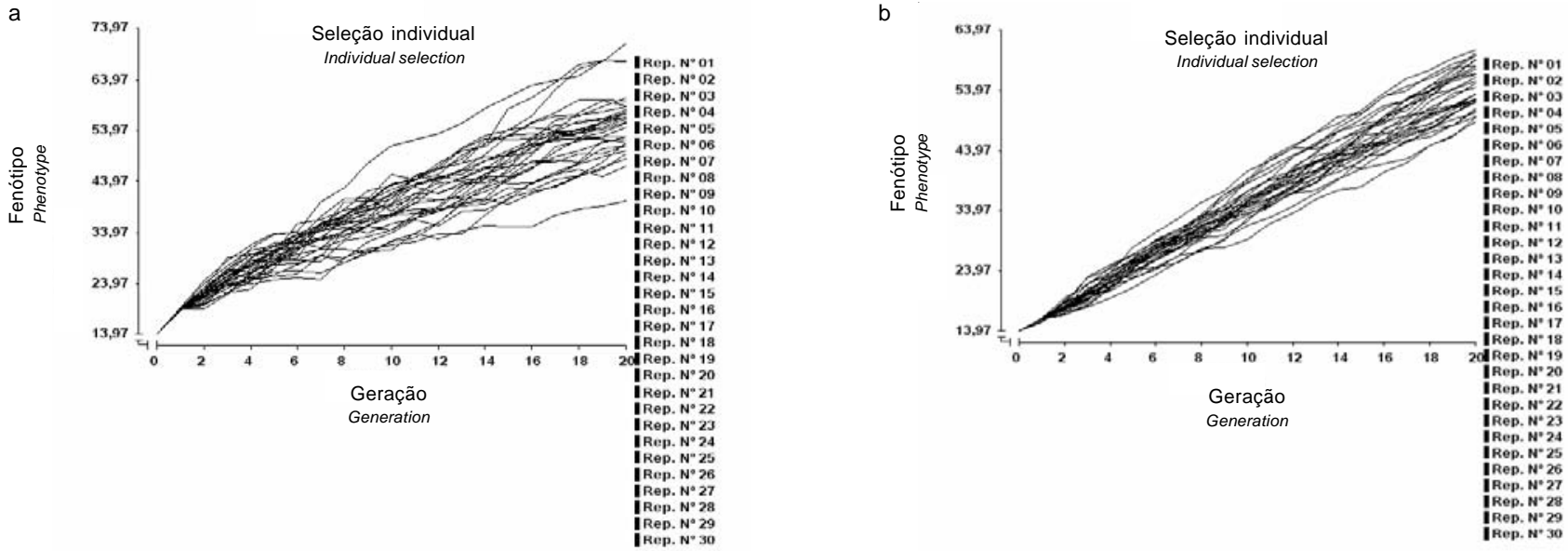

Figura 3 - Valores fenotípicos observados utilizando-se SI com exclusão de irmãos completos e meio-irmãos (EICMI), para populações com tamanho efetivo 1 e 2 (a e b), respectivamente.

Figure 3 - Observed phenotypic values using SI with exclusion of full-sibs and half-sibs; (EFSHS), for 1 and 2 effective size of the populations (a e b), respectively.
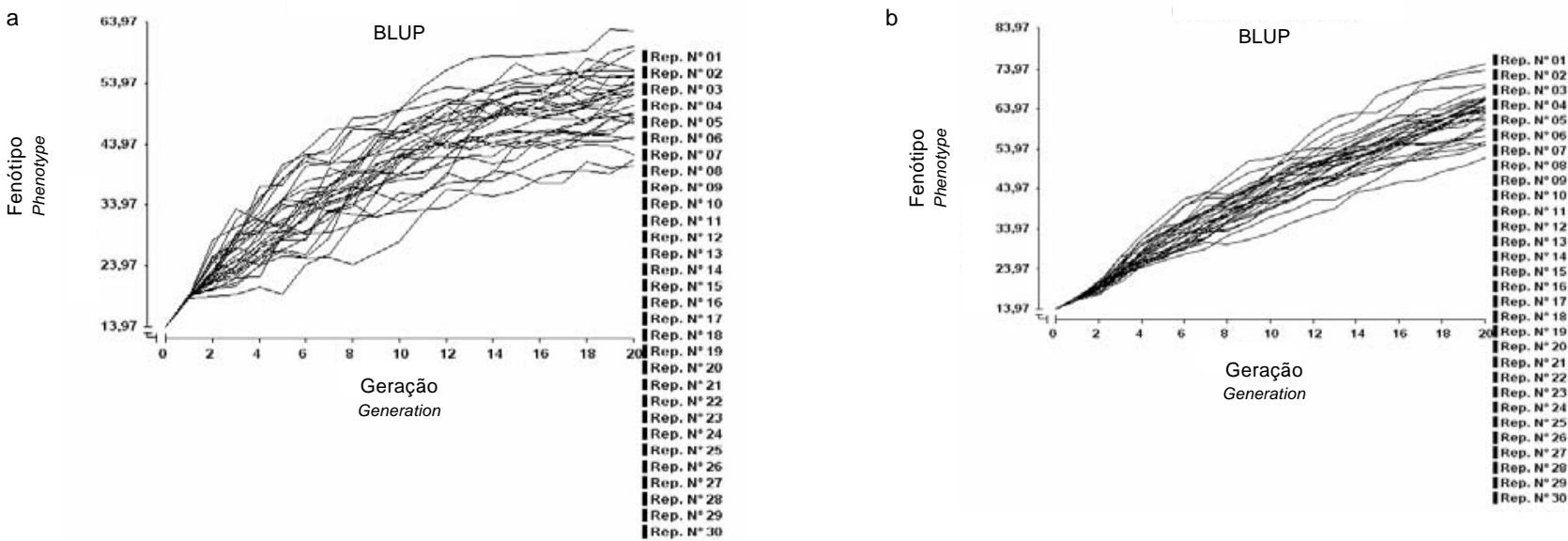

Figura 4 - Valores fenotípicos observados utilizando-se BLUP com reprodutores acasalados aleatoriamente (RAA), para populações com tamanho efetivo 1 e 2 (a e b), respectivamente.

Figure 4 - Observed phenotypic values using BLUP with random mating of reproducers (RM), for 1 and 2 effective size of the populations (a e b), respectively. 


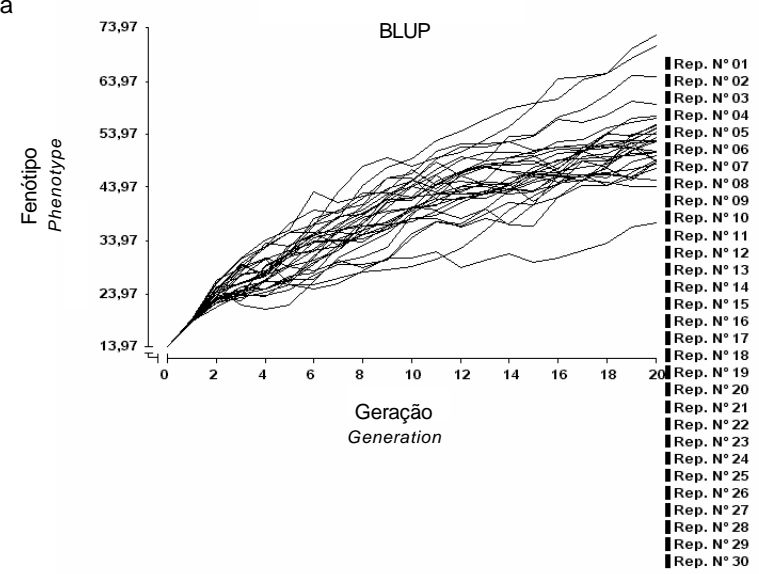

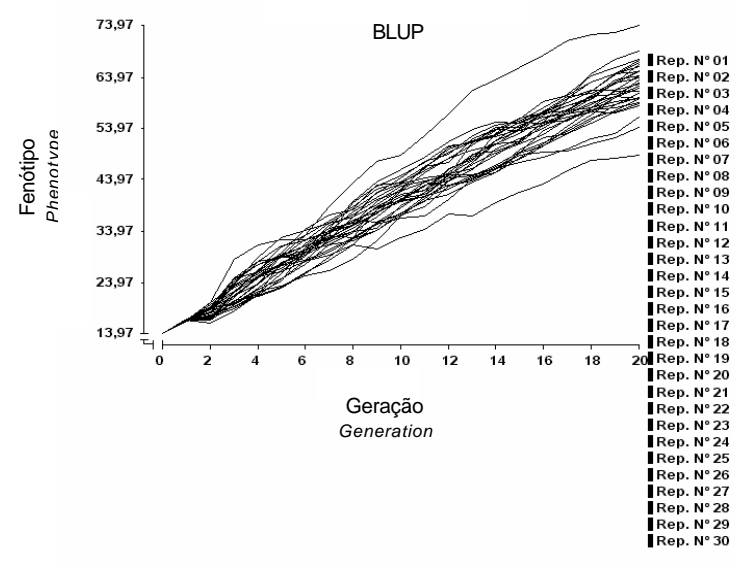

Figura 5 - Valores fenotípicos observados utilizando-se BLUP com exclusão de irmãos completos (EIC), para populações com tamanho efetivo 1 e 2 ( $a$ e b), respectivamente.

Figure 5 - Observed phenotypic values using BLUP with exclusion of full-sibs; (EFS), for 1 and 2 effective size of the populations (a e b), respectively.

a

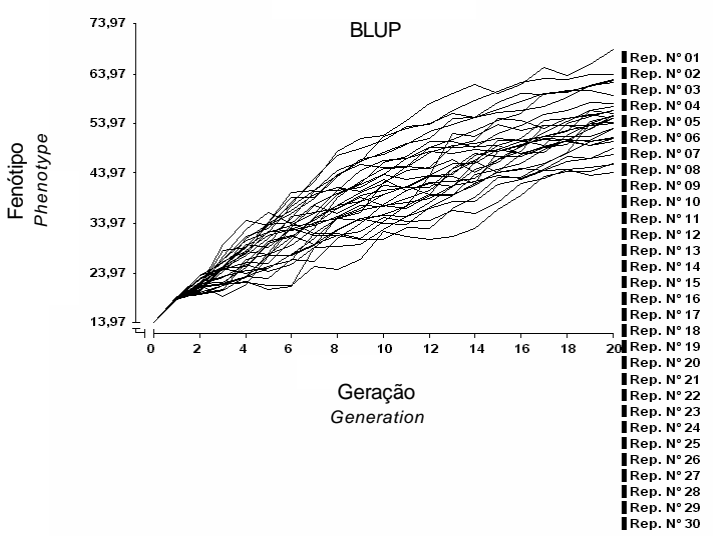

b

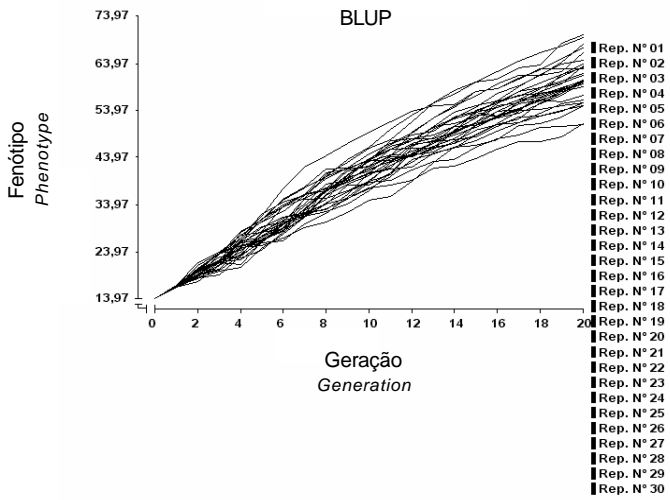

Figura 6 - Valores fenotípicos observados utilizando-se BLUP com exclusão de irmãos completos e meio-irmãos (EICMI), para populações com tamanho efetivo 1 e 2 (a e b), respectivamente.

Figure 6 - Observed phenotypic values using BLUP with exclusion of full-sibs and half-sibs; (EFSHS), for 1 and 2 effective size of the populations (a e b), respectively.

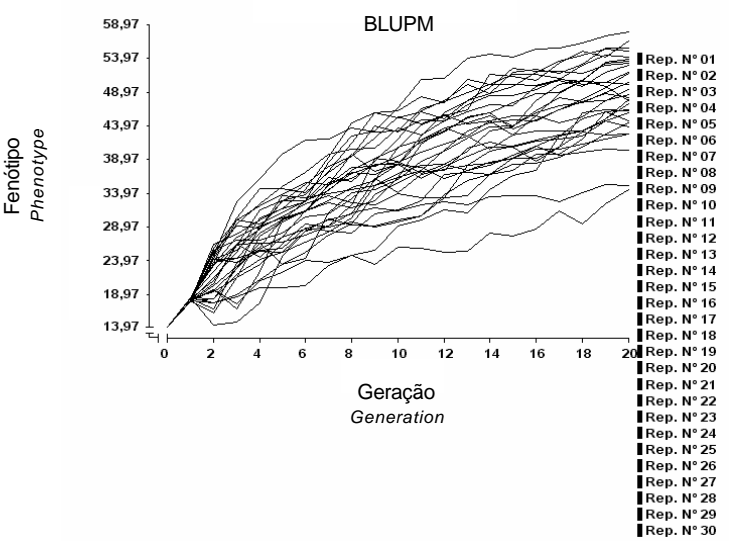

b

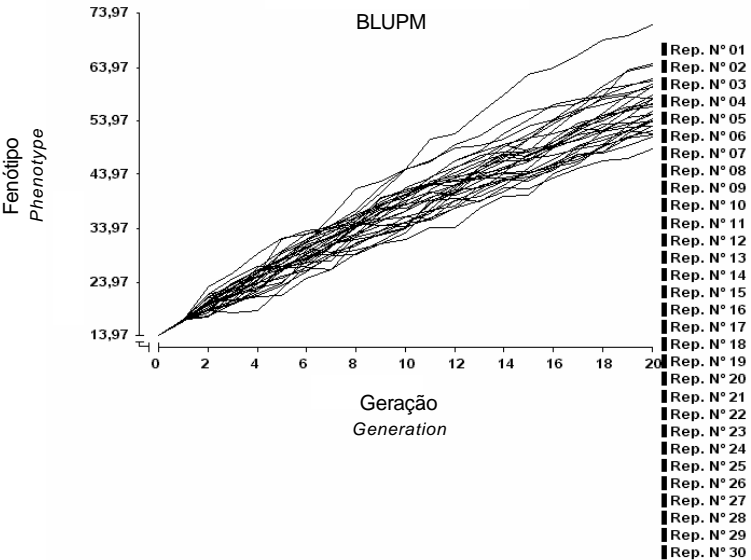

Figura 7 - Valores fenotípicos observados utilizando-se BLUPM com reprodutores acasalados aleatoriamente (RAA), para populações com tamanho efetivo 1 e 2 ( $a$ e b), respectivamente.

Figure 7 - Observed phenotypic values using BLUPM with random mating of reproducers (RM), for 1 and 2 effective size of the populations (a e b), respectively. 
a

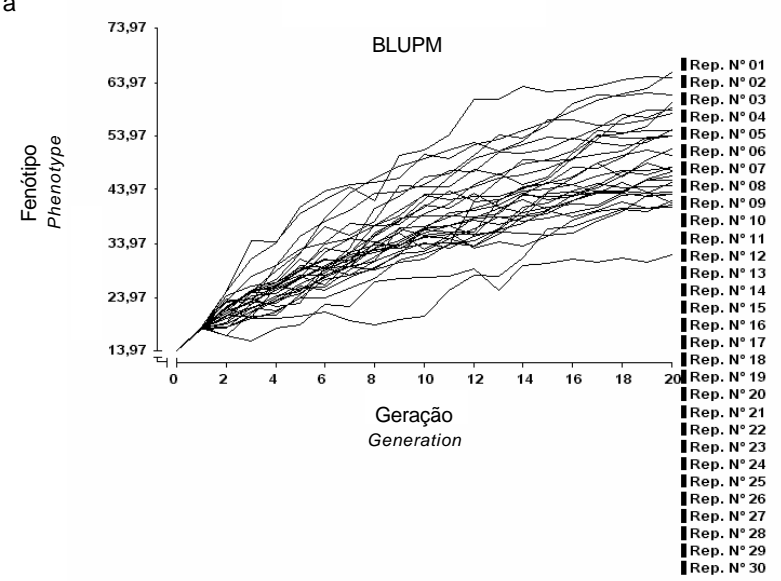

b

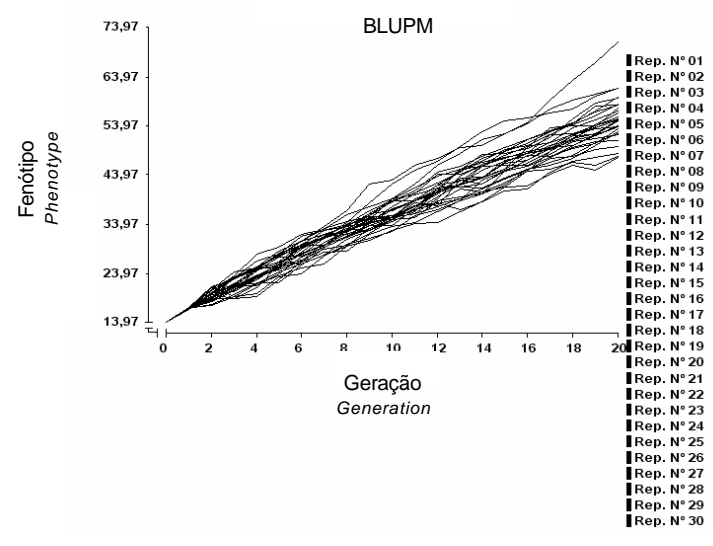

Figura 8 - Valores fenotípicos observados utilizando-se BLUPM com exclusão de irmãos completos (EIC), para populações com tamanho efetivo 1 e 2 (a e b), respectivamente.

Figure 8 - Observed phenotypic values using BLUPM with exclusion of full-sibs; (EFS), for 1 and 2 effective size of the populations (a e b), respectively.

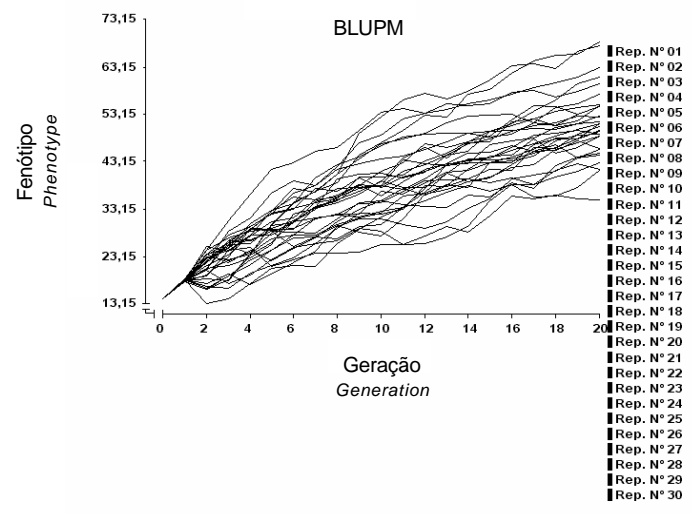

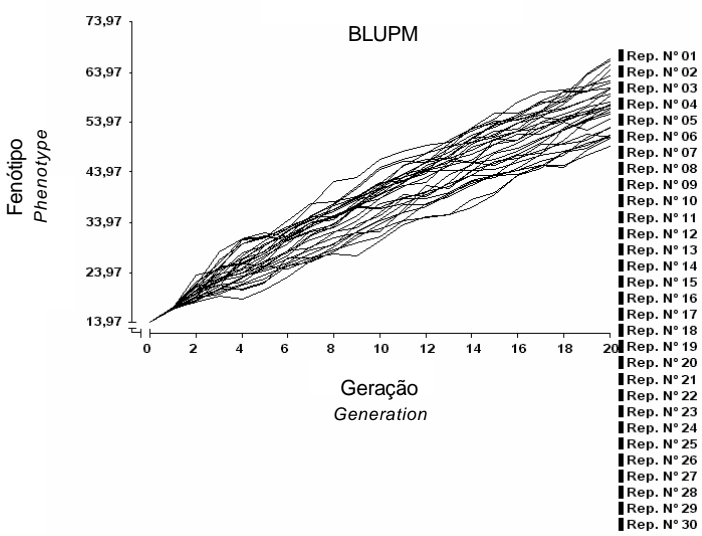

Figura 9 - Valores fenotípicos observados utilizando-se BLUPM com exclusão de irmãos completos e meio-irmãos (EICMI), para populações com tamanho efetivo 1 e 2 (a e b), respectivamente.

Figure 9 - Observed phenotypic values using BLUPM with exclusion of full-sibs and half-sibs; (EFSHS), for 1 and 2 effective size of the populations (a e b), respectively.

Considerando os resultados, ressalta-se que, em diferentes programas de melhoramento nos quais se utilizam pequenas populações, mesmo que seja utilizado o mesmo material genético e que todos os demais procedimentos sejam semelhantes, os resultados ao longo de várias gerações podem ser completamente diferentes, em decorrência da possibilidade de grande oscilação genética. Isso pode possibilitar, por exemplo, que determinado programa de melhoramento atinja o objetivo em um número de gerações de seleção diferente de outro. Há também a possibilidade de que os objetivos não sejam atingidos mesmo em muitas gerações.

As populações com TE1 e TE2 apresentavam as relações de 1 macho para 10 e 5 fêmeas, com total de 250 e 500 animais, respectivamente. Os resultados obtidos indicam a importância de se trabalhar com populações com tamanhos efetivos grandes (maior número de animais e relação macho-fêmea mais estreita), evitando-se a utilização de poucos machos para número grande de fêmeas, o que reduziria o tamanho efetivo da população. Esse aspecto foi discutido por Migglior \& Burnside (1995), em estudo com populações de gado de leite, no qual comprovaram que a utilização de alta intensidade de seleção, de técnicas reprodutivas que exploram mais intensivamente os animais (inseminação artificial, transferência de embriões, divisão de embriões e produção de embriões in vitro), de avaliações genéticas mais acuradas e de classificação global de reprodutores aumenta a endogamia e reduz o tamanho efetivo da população.

Estudos de simulação feitos por Belonsky \& Kennedy (1988) em população fechada dão suporte a esta hipótese. Um aumento na endogamia é esperado, em razão da classificação global dos reprodutores e do uso de poucos touros para melhorar os rebanhos, o que reduz o tamanho efetivo das populações sob seleção. Segundo Quinton et al. (1994), citado por Quinton \& Smith (1995), apenas o aumento do número de machos selecionados, que implica aumento do 
tamanho efetivo da população $\left(N_{e}\right)$, já seria suficiente para alterar as respostas esperadas para diferentes métodos de seleção propostos.

No Brasil, a redução do tamanho efetivo das populações também começa a ser bastante discutida. Faria et al. (2001a, b, c, d, e, f, g) calcularam o tamanho efetivo nas principais raças zebuínas e observaram que de 1979 a 1998 os tamanhos efetivos dessas populações foram significativamente reduzidos, em decorrência do uso intensivo de alguns reprodutores, refletindo no aumento da variância do número de progênie por reprodutor. Para a raça Nelore, grupamento genético que possui a maior população na pecuária nacional, os tamanhos efetivos da população nos períodos de 1979-1983, 1984-1988, 1989-1993 e 1994-1998 foram, respectivamente, $N_{e}=877$, 162, 71 e 34. Portanto, o $N_{e}$ para o último período é pequeno e pode ocasionar reduções nos ganhos genéticos.

A redução do tamanho efetivo pode ser atribuída a fatores como flutuações no tamanho populacional, desvios da razão de sexos de 1:1 e a diferenças no sucesso reprodutivo, o que pode ser observado com o aumento da variância do número de progênies. Goddard \& Smith (1990) sugerem tamanho efetivo mínimo de 40 por geração para maximizar o retorno econômico. Por outro lado, Meuwissen \& Woollians (1994) recomendam valores de 30 a 250 para se prevenir decréscimos no valor adaptativo.

Observou-se que a oscilação nos métodos de seleção foi maior para as populações submetidas à seleção pelos valores genéticos preditos pelo BLUP e BLUPM que pela SI. Entre o BLUP e o BLUPM, a oscilação foi um pouco maior no BLUPM, o que pode ser explicado pelo fato de a seleção com base nos valores genéticos obtidos pelos procedimentos estatísticos, baseados na metodologia dos modelos mistos (BLUP e BLUPM) poder conduzir a rápido aumento na taxa de endogamia e na porcentagem de alelos fixados desfavoravelmente, principalmente em populações de pequeno tamanho efetivo e para características de baixa herdabilidade.

Neste sentido, Muir (2000) afirma queo primeiro impacto da endogamiaéa perda de alelos de acordo com a oscilaçãogenética, que é diretamente proporcional aos níveis de endogamia.

Verrier et al. (1993) utilizaram simulação para verificar os efeitos da seleção a longo prazo em pequenas populações submetidas à seleção pelos valores genéticos preditos pelo BLUP sob modelo animal e observaram que, nas populações de pequeno tamanho, o efeito da oscilação foi maior, enquanto a variância genética decresceu rapidamente. Em contraste, em populações de tamanho maior, o efeito da oscilação não foi tão grande e a variância genética se manteve.

Euclydes(1996), Carneiro et al. (1999)e Cunha et al. (2003) observaram maiores taxas de endogamia e porcentagens de alelos fixados desfavoravelmente para a seleção utilizando os valores genéticos obtidos pelo BLUP em relação à seleção individual.

A endogamia é bom indicador da probabilidade de fixação de locos, isto é, o aumento na taxa de endogamia resulta em maior probabilidade de fixação de locos. Como observado por Belonsky \& Kennedy (1988), Kuhlers \& Kennedy (1992), Quinton et al. (1992), Jeyaruban et al. (1995), Quinton \& Smith (1995), a seleção por métodos baseados nos valores genéticos preditos pelo BLUP sob modelo animal tende a aumentar a endogamia, uma vez que os animais com maior valor genético tendem a ser mais relacionados.

Siegel \& Dunnington (1997) citam que o tamanho efetivo das populações e a intensidade de seleção influenciam não somente a taxa de mudança nas características sob seleção e correlacionadas, como também o tempo para se alcançar o limite de seleção.

Segundo Eisen (1975), a resposta à seleção aumenta com o incremento do $N_{e}$. A diferença na resposta à seleção, entre as linhas com diferentes $N_{e}$, para determinada intensidade de seleção, pode ser atribuída à redução endogâmica e à combinação de interações entre oscilação e seleção. Esse autor afirma ainda que a redução endogâmica e a oscilação genética são igualmente importantes para descrever as diferenças de $N_{e}$ entre as linhas na resposta total à seleção.

Entre os diferentes sistemas de acasalamento dos reprodutores selecionados (RAA, EIC e EICMI), não foram observadas grandes diferenças na oscilação genética ao longo das 20 gerações de seleção. Entretanto, pareceu haver tendência de redução da oscilação ao passar do sistema de acasalamento de RAA para EIC e deste para EICMI, o que pode estar relacionado ao fato de os sistemas de acasalamento EIC e EICMI proporcionarem taxas menores de endogamia e porcentagem de fixação de alelos desfavoráveis mais reduzida.

\section{Conclusões}

Em programas de melhoramento que utilizam pequenas populações sob seleção, os resultados podem ser influenciados pela oscilação genética, podendo ocasionar grandes variações nos ganhos genéticos.

A seleção de características quantitativas feita com base nos valores genéticos preditos pelo BLUP e pelo BLUPM apresentou maior oscilação genética, acentuada nas populações de menor tamanho efetivo.

\section{Literatura Citada}

AGGREY, S.E.; LIN, C.Y.; CHENG, K.M. Size of breeding populations required for selection programs. Theory Applied Genetic, v.91, p.553-556, 1995. 
BELONSKY, G.M.; KENNEDY, B.W. Selection on individual phenotype and best linear unbiased predictor of breeding value in a closed swine herd. Journal of Animal Science, v.66, p.1124-1131, 1988.

CARNEIRO, P.L.S.; EUCLYDES, R.F.; SILVA, M.A. et al. Efeito de erros de pedigree na seleção. Revista Brasileira de Zootecnia, v.28, n.2, p.269-274, 1999.

CARNEIRO, P.L.S; CORRÊIA, F.J.C; CARNEIRO JR., J.M. et al. Oscilação genética em diferente tamanhos de população sobre seleção. Arquivo Brasileiro de Medicina Veterinária e Zootecnia, v.54, n.1, p.84-92, 2002.

CUNHA, E.E.; EUCLYDES, R.F.; TORRES, R.A. et al. Efeito de tipos de acasalamentos e razões sexuais na seleção baseada no BLUP. Revista Brasileira de Zootecnia, v.32, n.6, p.12971303, 2003.

DEKKERS, J.C.M. Asymptotic response to selection on best linear unbiased predictors of breeding values. Animal Production, v.54, p.351-360, 1992.

EISEN, E.J. Population size and selection intensity effects on long term selection response in mice. Genetics, v.79, p.305-323, 1975.

EUCLYDES, R.F. Uso do sistema para simulação Genesys na avaliação de métodos de seleção Clássicos e associados a marcadores moleculares. Viçosa, MG: Universidade Federal de Viçosa, 1996. 149p. Tese (Doutorado em Zootecnia) Universidade Federal de Viçosa, 1996.

EUCLYDES, R.F.; GUIMARÃES, S.E.F. Associação dos métodos tradicionais de seleção à seleção assistida por marcadores moleculares. Revista Brasileira Reprodução Animal, v.21, n.3, p.89-96, 1997. (Anais do I Fórum Nacional de Equideocultura. XII Congresso Brasileiro de Reprodução Animal)

FALCONER, D.S. Introdução a genética quantitativa. SILVA, M.A.; SILVA, J.C. (Tradução). Viçosa, MG: Universidade Federal de Viçosa, 1981. 279p.

FARIA, F.J.C.; VERCESI FILHO, A.E.; MADALENA, F.E. et al. Intervalo de gerações e tamanho efetivo da população na raça Guzerá. In: REUNIÃO ANUAL DA SOCIEDADE BRASILEIRA DE ZOOTECNIA, 38., 2001, Piracicaba. Anais... Piracicaba: Sociedade Brasileira de Zootecnia, 2001a. p.480-481.

FARIA, F.J.C; VERCESI FILHO, A.E; MADALENA, F.E. et al. Intervalo de gerações e tamanho efetivo da população na raça Gir Mocho. In: REUNIÃO ANUAL DA SOCIEDADE BRASILEIRA DE ZOOTECNIA, 38., 2001, Piracicaba. Anais... Piracicaba: Sociedade Brasileira de Zootecnia, 2001b. p.482-483.

FARIA, F.J.C; VERCESI FILHO, A.E; MADALENA, F.E. et al. Intervalo de gerações e tamanho efetivo da população na raça Nelore Mocho. In: REUNIÃO ANUAL DA SOCIEDADE BRASILEIRA DE ZOOTECNIA, 38., 2001, Piracicaba. Anais... Piracicaba: Sociedade Brasileira de Zootecnia, 2001c. p.483-484.

FARIA, F.J.C; VERCESI FILHO, A.E; MADALENA, F.E.et al. Intervalo de gerações e tamanho efetivo da população na raça Gir. In: REUNIÃO ANUAL DA SOCIEDADE BRASILEIRA DE ZootecniA, 38., 2001, Piracicaba. Anais... Piracicaba: Sociedade Brasileira de Zootecnia, 2001d. p.484-485.

FARIA, F.J.C.; VERCESI FILHO, A.E; MADALENA, F.E.et al. Intervalo de gerações e tamanho efetivo da população na raça Indubrasil. In: REUNIÃO ANUAL DA SOCIEDADE BRASILEIRA DE ZOOTECNIA, 38., 2001, Piracicaba. Anais... Piracicaba: Sociedade Brasileira de Zootecnia, 2001e. p.485-486.

FARIA, F.J.C; VERCESI FILHO, A.E.; MADALENA, F.Eet al. Intervalo de gerações e tamanho efetivo da população na raça Nelore. In: REUNIÃO ANUAL DA SOCIEDADE BRASILEIRA DE ZOOTECNIA, 38., 2001, Piracicaba. Anais... Piracicaba: Sociedade Brasileira de Zootecnia, 2001f. p.486-487.
FARIA, F.J.C; VERCESI FILHO, A.E; MADALENA, F.E. et al. Intervalo de gerações e tamanho efetivo da população na raça Tabapuã. In: REUNIÃO ANUAL DA SOCIEDADE BRASILEIRA DE ZOOTECNIA, 38., 2001, Piracicaba. Anais... Piracicaba: Sociedade Brasileira de Zootecnia, 2001g. p.488-489.

GODDARD, M.G; SMITH, C. Optimum number of bull sires in dairy cattle breeding. Journal of Dairy Science, v.73, p.1113-1122, 1990.

JEYARUBAN, M.G.; GIBSON, J.P.; GOWE, R.S. Comparison of index selection and best linear unbiased prediction for simulated layer poultry data. Poultry Science, v.74, p.1566-1576, 1995.

KUHLERS, D.L; KENNEDY, B.W. Effect of culling on selection response using phenotypic selection or best linear unbiased prediction of breeding values in small, closed herds of swine. Journal of Animal Science, v.70, p.2338-2348, 1992.

MEUWISSEN, T.H.E; WOOLLIAMS, J.A. Effective sizes of livestock populations to prevent a decline in fitness. Theory Applied Genetic, v.89, p.1019-1026, 1994.

MIGLIOR, F; BURNSIDE, E.B. Inbreeding of Canadian Holstein cattle. Journal of Dairy Science, v.78, p.1163-1167, 1995.

MUIR, W.M. 2000. The interaction of selection intensity, inbreeding depression, and randon genetic drift on short and long-term response to selection: Results using finite locus and finite population size models incorporating directional dominance. Disponível em: http://www.asas.org. Acesso em: 25/04/2002.

QUINTON, M.; SMITH, C.; GODDARD, M.E. Comparison of selection methods at the same level of inbreeding. Journal of Animal Science, v.70, p.1060-1067, 1992.

QUINTON, M.; SMITH, C. Comparison of evaluation-selection system for maximizing genetic response at same level of inbreeding. Journal of Animal Science, v.73, n.8, p.2208-2212, 1995.

SIEGEL, P.B.; DUNNINGTON, E.A. Genetic selection strategiespopulation genetics. Poultry Science, v.76, p.1062-1065, 1997.

SORENSEN, D.A.; KENNEDY, B.W. The use of the relationship matrix to account for genetic drift variance in the analysis of genetic experiments. Theory Applied Genetic, v.6, p.217220, 1983.

VERRIER, E.; COLLEAU, J.J.; FOULLEY, J.L. Long-term effects of seletion based on the animal model BLUP in a finite population. Theory Applied Genetic, v.87, p.446-454, 1993.

VILLANUEVA, B.; WRAY, N.R.; THOMPSON, R. Prediction of asymptotic rates of response from selection on multiple traits using univariate and multivariate best linear unbiased predictors. Animal Production, v.57, p.1-13, 1993.

WEI, M.; CABALERO, A.; HILL, W.G. Selection response in finite populations. Genetics, v.144, p.1961-1974, 1996.

WRAY, N.R; HILL, W.G. Asymptotic rates of response from index selection. Animal Production, v.49, p.217-227, 1989.
Recebido: 11/11/04 Aprovado: 11/08/05 